\title{
Primary Tumor Resection is a Powerful Predictor of Long-term Outcome of Left-side Obstructive Colorectal Cancer
}

Hidejiro Kawahara ( $\nabla$ kawahide@outlook.jp )

Kokuritsu Byoin Kiko Nishisaitama Chuo Byoin https://orcid.org/0000-0002-8618-1556

Nobuo Omura

Kokuritsu Byoin Kiko Nishisaitama Chuo Byoin

Tadashi Akiba

Tokyo Jikeikai Ika Daigaku Fuzoku Kashiwa Byoin

\section{Research}

Keywords: obstructive colorectal cancer, primary tumor resection, predictor, cutcome, stoma

Posted Date: August 25th, 2020

DOI: https://doi.org/10.21203/rs.3.rs-64894/v1

License: (c) (1) This work is licensed under a Creative Commons Attribution 4.0 International License.

Read Full License 


\section{Abstract}

Aim

This study aimed to evaluate a long-term outcome predictor of left-side obstructive colorectal cancer.

Methods

Between 2001 and 2017, 40 patients with left-side obstructive colorectal cancer, 80 years of age or younger, who underwent emergency surgery at Kashiwa Hospital, Jikei University, were enrolled into this retrospective study. We compared 24 patients undergoing primary tumor resection with temporary colostomy with 16 patients undergoing only the creation of the decompression colostomy without primary tumor resection as the first operation.

\section{Results}

Three of 16 patients (19\%) who underwent only the creation of colostomy as the first operation could undergo the primary tumor resection as the secondary operation a month after the first operation. The other 13 patients who could not undergo the primary tumor resection received intensive chemotherapy consecutively. The 5-year survival rate of the patients with primary tumor resection as the first operation was $56.5 \%$, whereas that of patients with only the creation of colostomy as the first operation was $18.8 \%$ $(p<0.001)$. The fact that the primary tumor resection could not be performed was an independent risk factor by multivariate analyses $(p=0.002)$.

\section{Conclusion}

Primary tumor resection is a powerful predictor of long-term outcome of left-side obstructive colorectal cancer.

\section{Introduction}

Intestinal obstruction often occurs in patients with advanced colorectal cancer. Patients with acute leftside colonic obstruction have traditionally undergone emergency surgery that often requires the creation of a stoma, and approximately $40 \%$ of them will never have their stoma reversed [1-3]. Those with a permanent colostomy have a lower health-related quality of life. Stent placement can not only relieve the tumor obstruction but also buy sufficient time for intestinal preparation [4-7]. Per anus ileus catheterization can also temporarily relieve part of the intestinal obstruction and buy time for preoperative preparation $[8,9]$. Those procedures have important roles as a bridge to surgery. However, a predictor of the long-term outcome of left-side obstructive colorectal cancer has not been elucidated.

\section{Methods}


The Ethics Committee for Biomedical Research of the Jikei Institutional Review Board approved the protocol of this study [30-221(9242)]. Between 2001 and 2017, 40 patients with left-side obstructive colorectal cancer, 80 years of age or younger, who underwent emergency operation at Kashiwa Hospital, Jikei University, were enrolled into this retrospective study. They consisted of 24 patients undergoing primary tumor resection with temporary colostomy, so called Hartmann's operation, and 16 patients undergoing only the creation of the decompression colostomy without primary tumor resection as the first operation. The medical records of all patients were reviewed and classified according to the Japanese Classification of Colorectal Carcinoma [10].

\section{Treatment after the first surgery}

The primary tumor resection group $(n=24)$ received oxaliplatin combination intensive chemotherapy, such as infusional 5-fluorouracil and folinic acid plus oxaliplatin (FOLFOX), S-1 (Taiho Pharmaceuticals Co. Ltd, Tokyo, Japan) plus oxaliplatin (SOX), capecitabine plus oxaliplatin (CapeOX), for 6 months. After the chemotherapy, the patients without recurrence underwent intestinal reconstruction with closure of the colostomy. The other patients with recurrence received intensive chemotherapy consecutively according to the Japanese Society for Cancer of the Colon and Rectum guidelines [11] without closure of the colostomy.

Conversely, 16 patients with only the creation of the decompression colostomy as the first operation were scheduled to undergo the secondary operation, primary tumor resection, a month after the first operation. The patients who could not undergo the secondary operation received intensive chemotherapy consecutively according to the guidelines [11]. The patients who could undergo the secondary operation also received intensive chemotherapy consecutively according to the guidelines [11] after the secondary operation.

\section{Treatment schedule}

All patients were followed for 5 years; during this period, physical examinations, routine blood analyses, and serum carcinoembryonic antigen (CEA) measurements were conducted every two months after surgery. CT was performed every 2 months or when a patient's serum CEA value was higher than the normal level of $5.0 \mathrm{ng} / \mathrm{ml}$. Colonoscopy was performed every year or when a stool sample was positive for blood. Positron emission tomography (PET) or PET/CT was occasionally employed to detect occult metastasis for patients who had equivocal conventional imaging studies.

\section{Statistical analysis}

Continuous variables are expressed as the mean and range. The Wilcoxon rank-sum test was used for the comparison of continuous variables, and a chi-squared test was used for the comparison of categorical data. DFS after surgery was examined by the Kaplan-Meier method and by log-rank analysis. Variables affecting postoperative survival were analyzed using the Cox proportional hazards regression. A $p$-value of less than 0.05 indicated significance. All data were analyzed with IBM SPSS Statistics, version 24.0 (IBM Japan, Ltd, Tokyo, Japan). 


\section{Results}

Comparison of characteristics between the primary tumor resection group and only the creation of the decompression colostomy group in the first operation (Table 1)

Significant differences were identified in mean surgical time, mean blood loss, mean postoperative hospital stay, stage, and metastatic site, while significant differences were not found in mean age, gender, and primary tumor location. All 24 patients undergoing primary tumor resection with temporary colostomy could undergo intestinal reconstruction with closure of the colostomy after the intensive chemotherapy. Three of 16 patients $(19 \%)$ who underwent only the creation of the decompression colostomy as the first operation could undergo the primary tumor resection as the secondary operation a month after the first operation. The other 13 patients who could not undergo the primary tumor resection received intensive chemotherapy consecutively according to the guidelines [11].

Comparison of the 5-year survival rate between the primary tumor resection group and only the creation of the decompression colostomy group in the first operation

The 5-year survival rate of the 24 patients who underwent the primary tumor resection as the first operation was $56.5 \%$, whereas that of the 16 patients who underwent only the creation of the decompression colostomy was $18.8 \%$, and the difference was significant $(p<0.001)$ (Fig. 1).

\section{Cox proportional hazards regression for postoperative survival}

To determine the variables affecting postoperative survival, 5 variables (age, gender, stage, primary tumor location, and primary tumor resection) were analyzed using the Cox proportional hazards regression. Stage $(p=0.001)$ and primary tumor resection $(p=0.002)$ were identified as independent contributing factors to postoperative recurrence (Table 2).

\section{Discussion}

In the best supportive care without any systemic therapy, the median survival time of patients with unresectable colorectal cancer has been reported to be approximately 8 months [12]. Although recent systemic therapy has extended MST to approximately 30 months [13-15], unresectable colorectal cancer remains difficult to cure. The purpose of systemic therapy is to prolong the survival time and control symptoms by delaying tumor progression. Initially, unresectable colorectal cancer may become resectable after successful systemic therapy. Randomized controlled trials involving PS 0-2 patients have shown that systemic therapy is associated with a significantly longer survival time than BSC without any systemic therapy $[12,16]$.

In this study, 3 of 16 patients (19\%) who underwent only the creation of the decompression colostomy as the first operation could undergo primary tumor resection as the secondary operation a month after the 
first operation. The overall survival times of the patients without the primary tumor resection were within 14 months after the first operation if intensive chemotherapy was performed. The fact that the primary tumor resection could not be performed was an independent risk factor by multivariate analysis $(p=$ 0.002). When patients with left-side obstructive colorectal cancer are encountered, we should first evaluate the possibility of primary tumor resection.

In conclusion, primary tumor resection is a powerful predictor of long-term outcome of left-side obstructive colorectal cancer.

\section{Abbreviations}

FOLFOX: Infusional 5-fluorouracil and folinic acid plus oxaliplatin; SOX:S-1 plus oxaliplatin; CapeOX:Capecitabine plus oxaliplatin; CEA:Serum carcinoembryonic antigen; PET:Positron emission tomography.

\section{Declarations}

\section{Ethics approval and consent to participate}

The Ethics Committee for Biomedical Research of the Jikei Institutional Review Board approved the protocol of this study [30-221(9242)]. Individual patient consent is not required.

\section{Consent for publication}

There is no use of details, images, or videos relating to an individual person.

\section{Availability of data and materials}

All data generated or analysed during this study are included in this published article.

\section{Competing interests}

The authors declare no competing interests.

\section{Funding}

None.

\section{Authors' contributions}

HK and NO performed operation. All authors analyzed and interpreted the patient data, and have been involved in drafting the manuscript. TA had given final approval of the version to be published. All 


\section{Acknowledgements}

None.

\section{References}

1. Deans GT, Krukowski ZH, Irwin ST. Malignant obstruction of the left colon. Br J Surg. 1994;81(9):1270-6.

2. Watt AM, Faragher IG, Griffın TT, Rieger NA, Maddern GJ. Self-expanding metallic stents for relieving malignant colorectal obstruction: a systematic review. Ann Surg. 2007;246(1):24-30.

3. Kim JS, Hur H, Min BS, Sohn SK, Cho CH, Kim NK. Oncologic outcomes of self-expanding metallic stent insertion as a bridge to surgery in the management of left-sided colon cancer obstruction: comparison with nonobstructing elective surgery. World J Surg. 2009;33(6):1281-6.

4. Meisner S, González-Huix F, Vandervoort JG, Repici A, Xinopoulos D, Grund KE, Goldberg P, Registry Group TW. Self-Expanding Metal Stenting for Palliation of Patients with Malignant Colonic Obstruction: Effectiveness and Efficacy on 255 Patients with 12-Month's Follow-up. Gastroenterol Res Pract. 2012;20:296-347.

5. Buis Cl, Bosker RJ, ter Borg F, de Noo ME, Liem MS. Acute obstructive colon carcinoma and liver metastases: how to treat? Ned Tijdschr Geneeskd. 2012;156(43):A4817.

6. Zhang Y, Shi J, Shi B, Song CY, Xie WF, Chen YX. Self-expanding metallic stent as a bridge to surgery versus emergency surgery for obstructive colorectal cancer: a meta-analysis. Surg Endosc. 2012;26:110-9.

7. Lee HJ, Hong SP, Cheon JH, Kim TI, Min BS, Kim NK, Kim WH. Long-term outcome of palliative therapy for malignant colorectal obstruction in patients with unresectable metastatic colorectal cancers: endoscopic stenting versus surgery. Gastrointest Endosc. 2011;73(3):535-42.

8. Hotta T, Takifuji K, Kobayashi Y, Tabuse K, Shimada K, Maeda T, Nakatani Y, Fukiage O, Yamaue H. Management of obstructive colorectal cancer: evaluation of preoperative bowel decompression using ileus tube drainage. Surg Today. 2012;42(12):1154-64.

9. Zhang N, Zhou ZL, Xie JL. Application of transanal ileus tube in acute obstructive left-sided colorectal cancer. Int J Clin Exp Med. 2015;8(8):14024-9.

10. Japanese Society for Cancer of the Colon and Rectum. Japanese Classification of Colorectal Carcinoma, 2nd English edn. Tokyo:Kanehara Co. Ltd; 2009.

11. Watanabe T, Itabashi M, Shimada Y, et al. Japanese Society for Cancer of the Colon and Rectum (JSCCR) guidelines 2010 for the treatment of colorectal cancer. Int J Clin Oncol. 2012;17(1):1-29.

12. Simmonds PC. Palliative chemotherapy for advanced colo-rectal cancer: systematic review and meta-analysis. Colorectal Cancer Collaborative Group BMJ. 2000;321:531-5. 
13. Yamada $Y$, Takahari D, Matsumoto $H$, et al. Leucovorin, fluorouracil, and oxaliplatin plus bevacizumab versus $\mathrm{S}-1$ and oxaliplatin plus bevacizumab in patients with metastatic colorectal cancer (SOFT): an open-label, non-inferiority, randomised phase 3 trial. Lancet Oncol. 2013;14(13):1278-86.

14. Loupakis F, Cremolini C, Masi G, et al. Initial therapy with FOLFOXIRI and bevacizumab for metastatic colorectal cancer. N Engl J Med. 2014;371(17):1609-18.

15. Yamazaki K, Nagase M, Tamagawa $\mathrm{H}$, et al. Randomized phase III study of bevacizumab plus FOLFIRI and bevacizumab plus mFOLFOX6 as first-line treatment for patients with metastatic colorectal cancer (WJOG4407G). Ann Oncol. 2016;27(8):1539-46.

16. Cunningham D, Pyrhönen $S$, James RD, et al. Randomised trial of irinotecan plus supportive care versus supportive care alone after fluorouracil failure for patients with metastatic colorectal cancer. Lancet. 1998;31(9138):1413-8. 352(.

\section{Tables}

Due to technical limitations, tables are only available as a download in the Supplemental Files section.

\section{Figures}




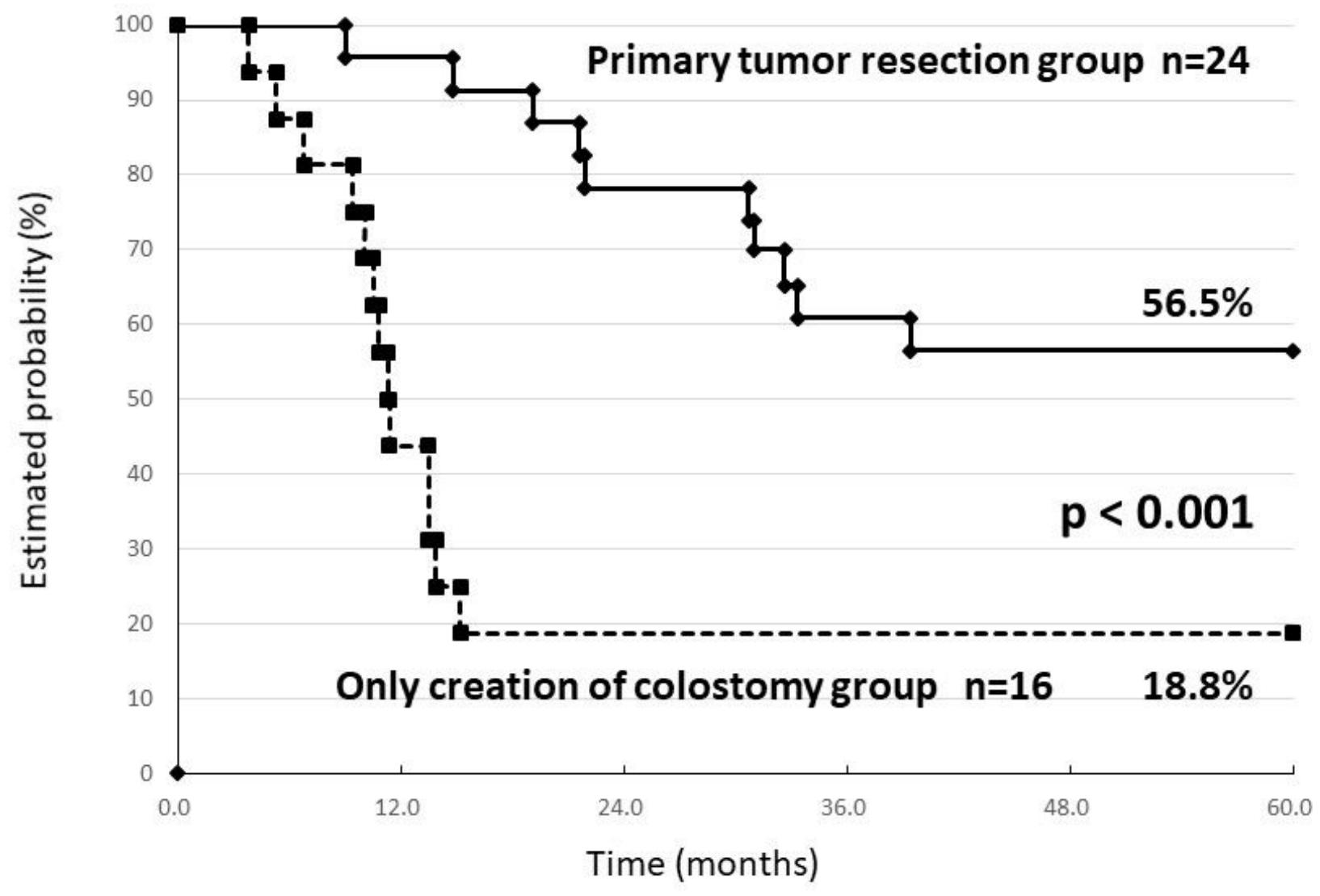

Figure 1

Comparison of the 5-year survival rate between the primary tumor resection group and only the creation of the decompression colostomy group in the first operation.

\section{Supplementary Files}

This is a list of supplementary files associated with this preprint. Click to download.

- Table2.xlsx

- Table1.xlsx 